\title{
Talar-tarsal Stabilisation: Goals and Initial Outcomes
}

\author{
Peter Stevens ${ }^{1}$, Alex Lancaster ${ }^{2}$, Ansab Khwaja ${ }^{3}$
}

\begin{abstract}
Background: While surgical stabilisation of the subtalar joint (arthroeresis) in children remains controversial in the USA, it is widely practised worldwide, with reportedly good outcomes. We are presenting a series of patients who met our criteria for calcaneal lengthening, but whose parents chose the less invasive option of talo-tarsal stabilisation (TTS). The goal of this surgery was to forestall or prevent hindfoot osteotomy. Materials and methods: With IRB approval, we conducted this retrospective review of 32 patients ( $60 \mathrm{ft}$ ), who underwent TTS for flexible planovalgus deformity and had a minimum of 1-year follow-up. The aetiology was idiopathic for the majority, with a few being neurogenic or syndromic. The age range was $6-15$ years; the younger patients had neuromuscular aetiology or underlying syndromes. Concomitant procedures included percutaneous Achilles lengthening ( $33 \mathrm{ft})$, Kidner ( $9 \mathrm{ft}$ ) and guided growth for ankle valgus (2).

Results: In the early post-immobilisation phase, peroneal spasm occurred in four patients ( $6 \mathrm{ft}$ ). This resolved with Botox injection in the peroneus brevis in three patients and required transfer of the peroneus brevis to the peroneus longus in one patient. At follow-up, ranging from 1 to 4.5 years, 50 implants (83.4\%) were retained and the patients reported satisfactory outcomes. Henceforth, those patients will be monitored on a p.r.n. basis. Due to lingering discomfort, implants were repositioned in one and removed in five patients $(10 \mathrm{ft}=16.6 \%)$. Upon further follow-up, these patients have not manifested recurrent deformity. Therefore, subsequent salvage by osteotomy and/or lengthening of the calcaneus has not been necessary.

Conclusion: TTS for the symptomatic flatfoot, combined with other procedures as indicated, offers advantages over the currently more accepted methods of medial shift osteotomy or calcaneal lengthening. The outcome at 1 year is a good forecast of whether or not further treatment will be required. This is a simpler and preferred option as compared to other methods of surgical management and, in our experience, has obviated the need for osteotomy or lengthening of the calcaneus.

Level of evidence: IV retrospective case series.

Keywords: Arthroeresis, Flatfeet, Planovalgus foot, Subtalar instability.

Strategies in Trauma and Limb Reconstruction (2021): 10.5005/jp-journals-10080-1538
\end{abstract}

\section{INTRODUCTION}

While there is no consensus, the most popular techniques for the surgical management of painful flatfeet in the growing child in the USA are either calcaneal lengthening or medial shift calcaneal osteotomy. These procedures are typically reserved for adolescents. While surgical stabilisation of the subtalar joint (arthroeresis) in children and adolescents remains controversial in the USA, it is widely practised worldwide, with good reported outcomes. Our purpose is to present a series of patients who either met our criteria for, or would eventually warrant, calcaneal lengthening. Upon discussion of the alternatives, their parents chose the less invasive option of talo-tarsal stabilisation (TTS). In particular, we wanted to assess the post-operative incidence of untoward outcomes, namely peroneal spasm or painful loosening of the implant, that usually manifest within the first year postoperatively and discuss the management of these problems.

\section{Materials and Methods}

With the requisite IRB approval, we undertook this retrospective review of 32 patients who underwent TTS to correct planovalgus deformity in $60 \mathrm{ft}$. All procedures were performed by the senior author, using the same design implant. There were 15 females and 17 males, ranging in age from 6 to 15 years at the time of surgery. While the youngest patients had neuromuscular issues or underlying syndromes, the aetiology for the vast majority was idiopathic. Inclusion criteria included any patient with flexible pronation/

\footnotetext{
1,2 Department of Orthopedics, University of Utah, Salt Lake City, Utah, United States of America

${ }^{3}$ Department of Orthopedics, University of Arizona, Phoenix, Arizona, United States of America
}

Corresponding Author: Peter Stevens, Department of Orthopedics, University of Utah, Salt Lake City, Utah, United States, of America Phone: +801 330-3656, e-mail: peter.stevens@mac.com

How to cite this article: Stevens P, Lancaster A, Khwaja A. Talar-tarsal Stabilisation: Goals and Initial Outcomes. Strategies Trauma Limb Reconstr 2021;16(3):168-171.

Source of support: Nil

Conflict of interest: None

planovalgus deformity. All patients had a history of recurring activity-related foot or leg discomfort and shoe wear intolerance. Many had had prior conservative management, including orthotics and/or physical therapy. The clinical examination included the observation of the pronated appearance of the foot and ankle in stance and during gait. We included patients with concomitant and symptomatic accessory navicular, manifest by medial prominence and localised tenderness. We confirmed hindfoot flexibility and evaluated for Achilles contracture, with the hindfoot inverted and the knee fully extended. If the hindfoot is not mobile, one should suspect a tarsal coalition and obtain further imaging preoperatively. None of the patients in this series had tarsal coalition.

(-) The Author(s). 2021 Open Access This article is distributed under the terms of the Creative Commons Attribution-Non Commercial-share alike license (https://creativecommons.org/licenses/by-nc-sa/4.0/) which permits unrestricted distribution, and non-commercial reproduction in any medium, provided you give appropriate credit to the original author(s) and the source, provide a link to the Creative Commons license, and indicate if changes were made. If you remix, transform, or build upon the material, you must distribute your contributions under the same license as original. The Creative Commons Public Domain Dedication waiver (http://creativecommons.org/publicdomain/zero/1.0/) applies to the data made available in this article, unless otherwise stated. 


\section{Imaging}

Standard radiographs obtained preoperatively and at least 12 months postoperatively included weight-bearing AP and lateral views of both ankles and feet. In patients with unilateral involvement (rare), the normal side was used for comparison. Consistent positioning of the patient is important so as to avoid projectional distortion and inaccurate measurements. Regarding the AP of the ankles, the lateral distal tibial angle (LDTA) normally measures between $87^{\circ}$ and $90^{\circ}$. Standard measurements of foot alignment have been well-described in the literature. ${ }^{2}$ However, if there is ankle valgus, this will exaggerate the measured talocalcaneal angles. ${ }^{3}$ On the lateral view, the tibia must be vertical $(0)$ or forward $(+)$ in order to appreciate the hindfoot alignment during single limb stance and at push-off (Fig. 1). This will demonstrate the respective contributions of a tight tendo-achilles and midfoot laxity to the overall malalignment. Having confirmed positioning and proper technique, we proceeded to measure Meary's angle and the convergent talocalcaneal angle on the lateral view. On the AP foot projection, we measured the divergent talo-calcaneal angle. We also checked for radiographic evidence of an accessory navicular.

\section{Surgical Technique}

The same day surgery was performed under tourniquet control and fluoroscopic guidance. Through a $2-\mathrm{cm}$ oblique lateral incision following Langer's lines, the sinus tarsi were probed with a haemostat. The lateral talo-calcaneal ligament is cut with curved Mayo scissors, to allow for proper seating of the implant, and the guide pin inserted into the sinus tarsi, aiming just inferior to the tip of the medial malleolus. The cannulated 5 -mm sizing probe was fully seated into the sinus tarsi. This was increased by $1-\mathrm{mm}$ increments, inspecting the heel position relative to the calf, with the goal of achieving neutral alignment. Care should be taken to avoid overcorrection into hindfoot varus. The corresponding diameter Talex cannulated implant (Vilex Inc. McMinnville, Tennessee) (Fig. 2) was placed over the guide pin and seated such that the head abutted the lateral aspect of the talus, whereupon the guide pin was removed and the wound closed with 3.0 and 4.0 Monocryl. With the knee in extension, passive range of motion of the ankle was then assessed and, if this stopped short of neutral, a percutaneous lengthening of the Achilles was accomplished. Concomitant guided growth or Kidner procedures were undertaken as indicated (Fig. 1).

Postoperatively, the patients were placed in a walking cast for 4 weeks and, for those requiring Achilles lengthening, a 3D boot for an additional 4 weeks. Physical therapy was not required, nor were any post-immobilisation activity limitations imposed. Follow-up ranged from 1 to 4.5 years. If the patients went on to experience discomfort, stiffness (due to peroneal spasm) or limping postoperatively, then additional weight-bearing radiographs were obtained in order to assess the alignment of the foot as well as the position of the subtalar implant. Radiographs were repeated at 1 year; if the patient was fully functional, without limping or shoe wear complaints, then follow-up was offered on a p.r.n. basis.
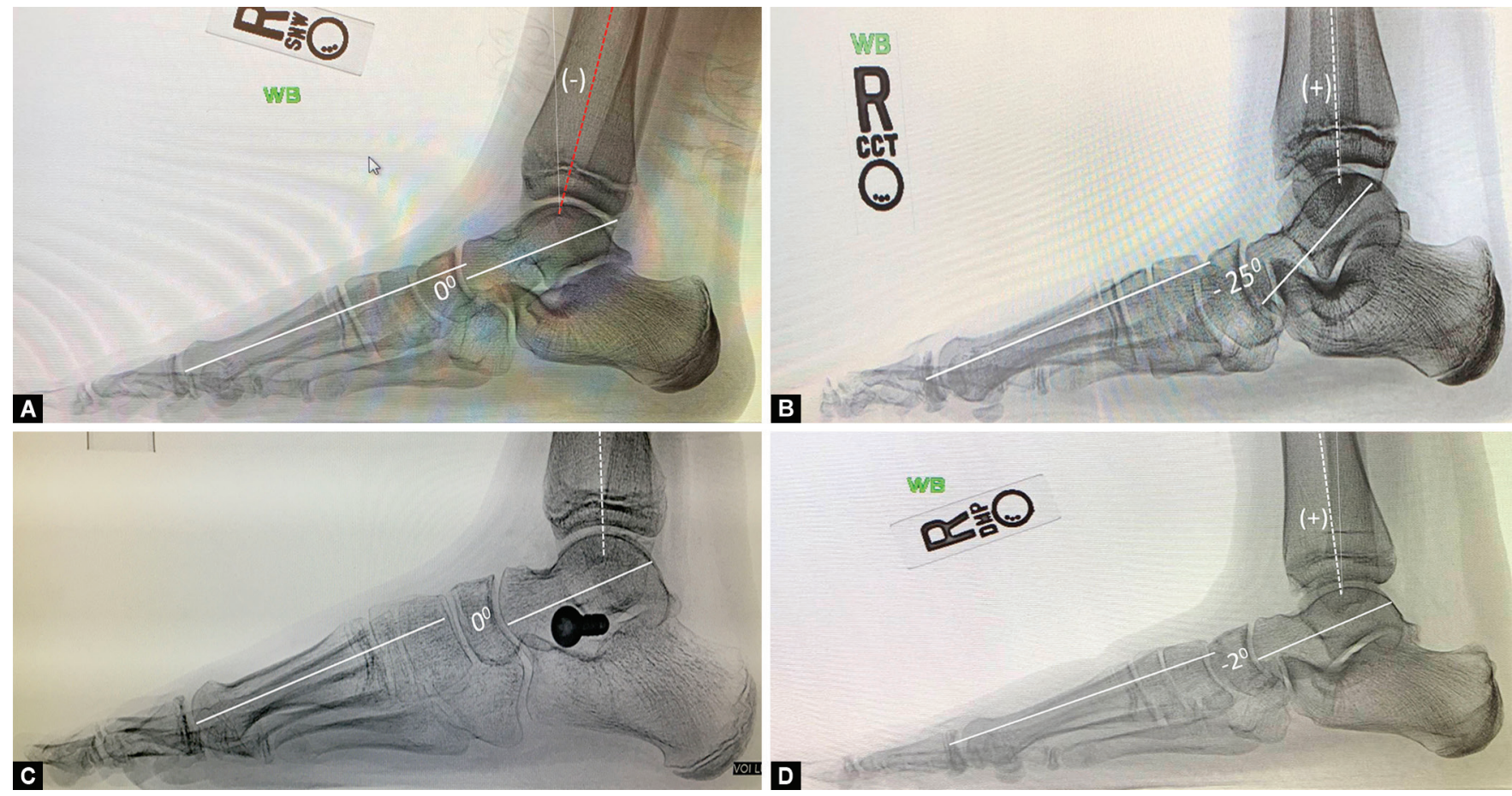

Figs 1 A to D: (A) Due to improper positioning, this weight-bearing lateral view of the right foot is inadequate and deceptive. It depicts the hindfoot alignment at heel strike and thus does not account for a tight tendo-achilles and ligamentous laxity in the hindfoot and midfoot. The tibia must be oriented vertically or slightly forward, in order to be true weight-bearing and deduce hindfoot alignment mid-stance; (B) Proper positioning (same patient/same day) with the tibia vertical provides a true weight-bearing view and illustrates the talo-tarsal instability that is manifest as a planovalgus foot deformity; (C) Hindfoot alignment has been restored with a subtalar implant, combined with percutaneous lengthening of the Achilles. The implant was left in situ for 16 months, whereupon it was removed because of local discomfort; (D) One year following removal, the alignment has been maintained and the patient is fully active without symptoms. There is no arthrosis of the subtalar joint 

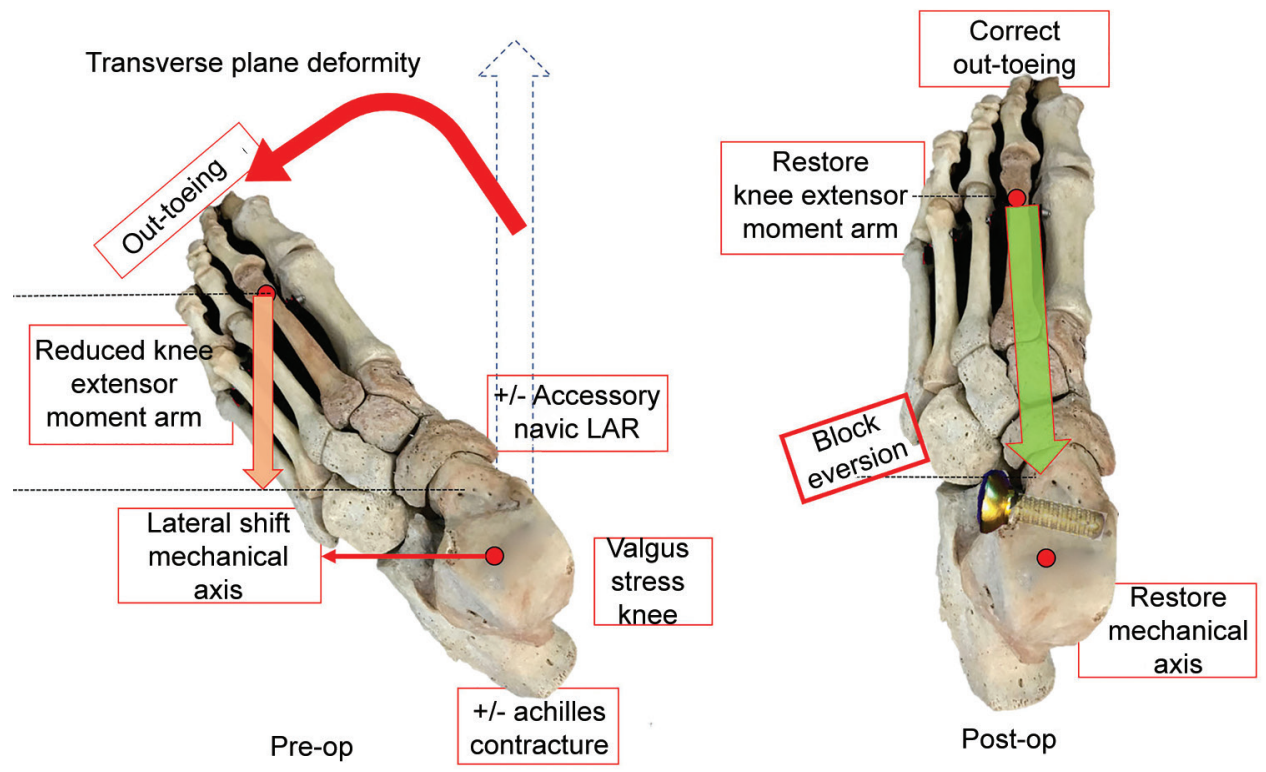

Fig. 2: Concept of TTS is illustrated in the transverse plane. Preoperatively, the ankle remains neutral during stance and gait. Meanwhile, the ligamentous laxity and the oblique plane of the subtalar joint yield to produce pronation of the hindfoot and obligate outward rotation of the midfoot and forefoot relative to the knee. The post-operative image depicts the transverse plane effect of TTS, which prevents the eversion/ outward rotation seen on the left. The mechanical axis is restored, relative to the ankle and the extensor moment at the knee improved by foot realignment and stabilisation

Table 1: Primary surgical procedures

\begin{tabular}{lccc}
\hline Index surgery & \# patients & \# feet & \% feet \\
\hline TTS + TAL & 16 & 29 & $48.3 \%$ \\
TTS alone & 11 & 21 & $36.7 \%$ \\
TTS + Kidner & 3 & 5 & $8.3 \%$ \\
TTS + TAL + Kidner & 2 & 4 & $6.7 \%$ \\
\hline
\end{tabular}

Table 2: Complications leading to subsequent interventions

\begin{tabular}{lccc}
\hline Problem & \# patients & \# feet & Remedy \\
\hline $\begin{array}{l}\text { Pain/c implant } \\
\text { loosening }\end{array}$ & 3 & $6(10 \%)$ & 2 reposition/4 removal \\
$\begin{array}{l}\text { Pain/s implant } \\
\text { loosening }\end{array}$ & 3 & $6(10 \%)$ & 6 removal \\
Peroneal spasm & 4 & $6(10 \%)$ & 5 Botox $/ 1$ brevis transfer \\
\hline
\end{tabular}

\section{Results}

There were no peri-operative complications. All patients resumed full activities upon cessation of immobilisation, which comprised of 1 month in casts $+/-$ an additional month of boots for those who had concomitant Achilles lengthening. During the ensuing months of follow-up, four patients $(6 \mathrm{ft})$ experienced discomfort and limping due to spasm in the peroneus brevis. This resolved with Botox injections in two patients. The third patient underwent transfer of the peroneus brevis to the peroneus longus (Table 1). The remainder progressed and improved without complaints.

Radiographs were repeated a year post-implantation. Our measurements were compared qualitatively, to ascertain restoration and maintenance of improved alignment. Ten of the implants were subsequently removed from five patients due to localised activity-related discomfort in the lateral hindfoot, with prominence and tenderness around the implants. This resolved their complaints, and none have manifest stiffness or arthrosis. Importantly, collapse and recurrent planovalgus have not ensued following implant removal. With subsequent follow-up, there has been no need to resort to salvage procedures such as medial shift calcaneal osteotomy or calcaneal lengthening (Table 2).

\section{Discussion}

By consensus, children who have flexible flatfeet, but manifest no symptoms, do not require orthopaedic intervention. Generally speaking, they have an excellent prognosis and may simply choose comfortable shoe wear, with or without arch support, according to their needs. However, a subset of the paediatric/adolescent population may present with pronated planovalgus feet due to talo-tarsal instability. They seek orthopaedic advice because of shoe intolerance and activity-related discomfort. Often, they have tried orthotics, physical therapy and NSAIDs without perceived benefit. The underlying aetiology may be idiopathic, neuromuscular or due to a syndrome. If their symptoms are unremitting and compromising their activities and lifestyle, surgical attention may be warranted. Consensus is lacking, but the traditional options have included lateral column lengthening with allograft vs medial shift osteotomy of the calcaneus. ${ }^{4-10}$ While TTS (arthroeresis) has become popular internationally, it is still not well-accepted amongst paediatric and foot/ankle surgeons in the USA, perhaps because the majority of the favourable references are currently in the podiatry or international literature..$^{11-18}$

In a previous prospective study, we found the outcomes and kinematics of arthroeresis and calcaneal lengthening to be comparable. ${ }^{19}$ In particular, we noted that the subtalar implants did not predispose to hindfoot stiffness or arthrosis. The validated Oxford questionnaire revealed that both patients and parents were highly satisfied with the TTS approach. post-operative return to normal lifestyle and activities was expedited because they do not have to delay weight-bearing and await bone graft 
incorporation. Furthermore, as opposed to a bone graft, if an implant fails, it may be removed without serious consequences. Based upon our experience, we have continued to favour TTS in lieu of the more invasive bony procedures. Finally, TTS is more appropriate than hindfoot osteotomy for younger children with neurogenic compromise or syndromes and pre-adolescents who have substantial tarsal growth and thus could experience recurrent deformity following osteotomy.

This review focused upon short-term results of a series of patients who underwent TTS in lieu of calcaneal lengthening. Our particular interest was to document the tolerance of the implants and discuss ways to mitigate the adverse outcomes. We found these to be comparatively uncommon and not difficult to manage. Peroneal spasm responded to Botox in three patients and required a tendon transfer in one. The majority $(85 \%)$ of patients remain asymptomatic and fully active with retained implants. In the remainder, implant removal resolved lingering discomfort while hindfoot correction was maintained; these patients have not required further treatment. We did not perform a cost comparison between TTS and osteotomy, but the aggregate cost, considering operative time, hospital stay, mobilisation, radiographs and ancillary factors including clinic visits, travel costs, time away from work and school, would undoubtedly favour the less invasive procedure. While an implant can always be repositioned or removed, a bone graft cannot. Based upon our experience, our strategy of primarily performing TTS in order to postpone or, more likely, prevent hindfoot osteotomy had been successful.

\section{References}

1. Paley D. Principles of deformity correction. New York, NY: Springer Berlin Heidelberg; 2003.

2. Davids JR, Gibson TW, Pugh LI. Quantitative segmental analysis of weightbearing radiographs of the foot and ankle for children: normal alignment. J Pediatr Orthop 2005;25(6):769-776. DOI: 10.1097/01. bpo.0000173244.74065.e4.

3. Stevens PM. Effect of ankle valgus on radiographic appearance of the hindfoot. J Pediatr Orthop 1988;8(2):184-186. PMID: 3350953.

4. Evans D. Calcaneovalgus deformity. J Bone Joint Surg Br 1975;57(3):270-278. PMID: 1171869.

5. Mosca VS. Calcaneal lengthening for valgus deformity of the hindfoot. Results in children who had severe, symptomatic flatfoot and skewfoot. J Bone Joint Surg 1995;77(4):500-512. DOI: 10.2106/00004623-199504000-00002.
6. Mosca VS. Flexible flatfoot in children and adolescents. J Child Orthop 2010;4(2):107-121. DOI: 10.1007/s11832-010-0239-9.

7. Rathjen KE, Mubarak SJ. Calcaneal-cuboid-cuneiform osteotomy for the correction of valgus foot deformities in children.J Pediatr Orthop 1998;18(6):775-782. PMID: 9821135.

8. Moraleda L, Salcedo M, Bastrom TP, et al. Comparison of the calcaneo-cuboid-cuneiform osteotomies and the calcaneal lengthening osteotomy in the surgical treatment of symptomatic flexible flatfoot. J Pediatr Orthop 2012;32(8):821-829. DOI: 10.1097/ BPO.0b013e3182648c74.

9. Andreacchio A. Lateral column lengthening as treatment for planovalgus foot deformity in ambulatory children with spastic cerebral palsy. J Pediatr Orthop 2007;27(3):364. DOI: 10.1097/ 01.bpb.0000248578.04241.75.

10. Adams SB Jr, Simpson AW, Pugh LI, et al. Calcaneocuboid joint subluxation after calcaneal lengthening for planovalgus foot deformity in children with cerebral palsy. J Pediatr Orthop 2009;29(2):170-174. DOI: 10.1097/BPO.0b013e3181982c33.

11. Soomekh D, Baravarian B. Pediatric and adult flatfoot reconstruction: subtalar arthroeresis versus realignment osteotomy surgical options. Clin Podiatr Med Surg 2006;23(4):695-708. DOI: 10.1016/ j.cpm.2006.08.003.

12. Metcalfe S, Bowling F, Reeves N. Subtalar arthroeresis in the management of pediatric flatfoot: a clinical review literature. Foot Ankle Int 2011;32(12):1127. DOI: 10.3113/FAl.2011.1127.

13. Retana P, Alvarez F, Viladot R. Subtalar arthroeresis in pediatric flatfoot reconstruction. Foot Ankle Clin 2010;15(2):323-335. DOI: 10.1016/ j.fcl.2010.01.001.

14. Cook E, Cook J, Basile P. Identifying risk factors in subtalar arthroeresis explanation: a propensity-matched analysis. J Foot Ankle Surg 2011;50(4):395-401. DOI: 10.1053/j.jfas.2011.03.019.

15. Bernasconi A, Lintz F, Sadile F. The role of arthroereisis of the subtalar joint for flatfoot in children and adults. EFORT Open Rev 2017;2(11):438-446. DOI: 10.1302/2058-5241.2.170009.

16. DePellegrin $M$, Moharamzadeh $D$, Strobl $M$, et al. Subtalar extraarticular screw arthroereisis (SESA) for the treatment of flexible flatfoot in children. J Child Othop 2014;8(6):479-487. DOI: 10.1007/ s11832-014-0619-7.

17. Shah N, Needleman R, Bokhari O, et al. Subtalar arthroereisis survey: the current practice patterns of members of the AOFAS. Foot Ankle Spec 2015;8(3):180-185. DOI: 10.1177/1938640015578514.

18. Vedantam R, Capelli AM, Schoenecker PL. Subtalar arthroeresis for the correction of planovalgus foot in children with neuromuscular disorders. J Pediatr Orthop 1998;18(3):294-298. PMID: 9600551.

19. Chong D, MacWilliams B, Hennessey T, et al. Prospective comparison of subtalar arthroeresis with lateral column lengthening for painful flatfeet. J Pediatr Orthop (Br) 2015;24(4):345-353. DOI: 10.1097/ BPB.0000000000000179. 